\title{
Intermédialités
}

Histoire et théorie des arts, des lettres et des techniques

Intermediality

History and Theory of the Arts, Literature and Technologies

\section{Sauts temporels et dé-placements}

\section{Le souvenir du travail dans les machines de vision de Farocki}

\section{Thomas Elsaesser}

Numéro 11, printemps 2008

Travailler (Harun Farocki)

Working (Harun Farocki)

URI : https://id.erudit.org/iderudit/037536ar

Aller au sommaire du numéro

Éditeur(s)

Centre de recherche sur l'intermédialite

ISSN

1705-8546 (imprimé)

1920-3136 (numérique)

Découvrir la revue

Citer cet article

Elsaesser, T. (2008). Sauts temporels et dé-placements : le souvenir du travail dans les machines de vision de Farocki. Intermédialités / Intermediality, (11), $35-51$

\section{Résumé de l'article}

Cet essai soutient que les films de Harun Farocki ont toujours oscillé entre les contraintes institutionnelles, d'une part, et les failles du cinéma indépendant (financé par l'État et la télévision) et des galeries d'art (commanditées), d'autre part. Cela dit, l'autorité morale et la crédibilité esthétique de ses films et de ses installations découlent d'une " critique " à double tranchant : ses oeuvres sont critiques parce que leur portée réflexive atteint même le réalisateur, et que la boucle de rétroaction (feedback) engage également l'artiste. Quiconque explore son travail/art découvre que Farocki est vraisemblablement l'un des rares réalisateurs contemporains à pouvoir contrecarrer le monde comme oeil machinique, en redonnant à l'oeil et la main, par moment, une puissance d'implication de soi et de solidarité. Conséquemment, toute l'actualité et l'urgence de l'oeuvre de Farocki tiennent dans un effort pour sauvegarder le cinéma de sa propre dialectique de mémoire et d'oubli, d'une évocation nostalgique d'une plénitude perdue et d'une auto-réflexivité moderne, en réinventant le " travail " comme " art ", et l'" art " comme " travail ".
Ce document est protégé par la loi sur le droit d'auteur. L'utilisation des services d'Érudit (y compris la reproduction) est assujettie à sa politique d'utilisation que vous pouvez consulter en ligne.

https://apropos.erudit.org/fr/usagers/politique-dutilisation/ 


\title{
Sauts temporels et dé-placements \\ Le souvenir du travail dans les machines de vision de Farocki
}

\author{
Thomas Elsaesser
}

\section{LA POST AVANT-GARDE : LE CINÉMA Au MUSÉE}

Quand on considère le sort actuel de l'avant-garde filmique des années 1960 et 1970, on est d'abord étonné que les films soient maintenant présentés dans les musées et les galeries d'art plutôt que sur un écran de cinéma. Évidemment, cet exode s'explique principalement par un problème de distribution : au cours des vingt dernières années, les lieux de diffusion des films «expérimentaux» ou non narratifs (salles de cinéma de répertoire, Programmkinos, cinémathèques) se sont faits plus rares et les projections moins rentables, sauf lors d'un événement spécial (projection commémorative ou rétrospective d'un artiste établi). Comme les films ne sont présentés que lors d'événements rétrospectifs (ce qui indique que le cinéma soit maintenant le lieu principal de notre mémoire collective), ni la rétrospective ni la commémoration n'engendrent de nouvelles productions, et par conséquent la question du financement explique aussi largement ce passage de la salle de cinéma au musée. La télévision, du moins en RFA, constituait la principale source de financement et le principal lieu de diffusion de l'avant-garde. Toutefois, cette source s'est elle aussi tarie durant les années 1980, en raison de la croissance de la télédiffusion commerciale. Une case horaire en fin de soirée n’a jamais pu rendre justice à des œuvres créées au fil des mois, voire des années. L'installation artistique offre ainsi une alternative: les créateurs ont trouvé un parrain en la personne du commissaire d'exposition, une autorité pour commander et conserver de nouvelles œuvres et pour conserver les anciennes. Une galerie ou un musée garantissent un meilleur temps de diffusion des œuvres. Ils garantissent la visite de personnes attentives, des visiteurs plutôt que des téléspectateurs (tv-viewers) qui ne feraient que regarder en passant d'une chaîne à l'autre, ou des spectateurs de cinéma qui ne chercheraient qu'un divertissement. 
Mais par-delà ces raisons évidentes pour présenter des films d'art au musée, quelles sont les conséquences de cette migration de la «post avant-garde »? Nous pouvons d'emblée tirer quelques conclusions, sans calculer dans le détail les gains et les pertes qui résultent de la «mort du cinéma [d'avant-garde] » et de sa renaissance dans l'installation artistique. Premièrement, au plan historique, les avant-gardes ont toujours résisté au monde de l'art, tout en dépendant néanmoins de son réseau institutionnel. Il s'agit d'une contradiction intrinsèque qui conditionne aussi la nouvelle union entre le film d'avant-garde et le réseau muséal et qui semble créer des impasses en ce qui concerne «l'original» et la «copie», «l'indépendance» et le «marché». Le film comme parangon de la « reproduction mécanique» se retrouve à présent pris en charge par les institutions vouées à la culture (et au culte) de l'œuvre authentique, dont l'unicité tient autant à l'aura qu'à la valeur marchande. Deuxièmement, le contraste entre la durée d'une œuvre (parfois de plusieurs heures: Jean-Luc Godard, Douglas Gordon, Ulrike Ottinger, Phil Collins) et la durée de la visite en galerie (quelques minutes devant un écran, un moniteur ou une installation) suscite des tensions productives. Ainsi, les répétitions, les boucles, les juxtapositions, les constructions en miroir et les formes sérielles prédominent sur des principes de structuration comme la trajectoire linéaire, narrative ou argumentative (Dan Graham, Matthias Müller, Christian Marclay, Martin Arnold). Troisièmement, l'installation permet de nouvelles manières de s'adresser directement au spectateur: par l'usage du son qui crée une présence singulière (de Jean-Marie Straub/Danielle Huillet à Janet Cardiff/George Bures Miller) ou par le recours à des juxtapositions d'écran qui obligent le regardeur à pallier le hiatus en fournissant son propre «trait d'union » (Isaac Julien, Stan Douglas, Chantal Akerman, Harun Farocki). Enfin, et d'une manière cruciale, la réflexivité et l'auto-référentialité programmatiques de l'œuvre d'art moderne sont à la fois prolongées et subverties par l'installation artistique ou par de nouvelles formes de performativité et d'implication qui intègrent parfois corporellement l'artiste (dans la tradition de Carolee Schneemann ou d'Yvonne Rainer), parfois même en le blessant (Marina Abramovic, Harun Farocki).

Pour le dire autrement, je pense que Harun Farocki (cinéaste et créateur de nombreuses installations) constitue un cas exemplaire pour comprendre cette migration: ses films ont toujours incarné les paramètres esquissés ci-dessus, et ses installations gardent certaines des caractéristiques essentielles de la création filmique, tant comme art «public» que comme une sorte «d'artisanat» ou de 
métier ${ }^{1}$. Le sous-titre de ce texte porte ainsi le terme «travail» (work), un mot qui renvoie à l'œuvre ainsi qu'à une façon possible de comprendre ses films et ses installations: comment l'histoire du travail et du labeur ainsi que la mémoire de l'œuvre ont à voir avec l'avenir de l'art comme concept, marchandise et artefact. Cela implique qu'il faille considérer la contradiction du «cinéma au musée » d'une manière légèrement différente de celle qui a été présentée précédemment. La contradiction des œuvres en galerie de Farocki tient autant à l'imperceptible dialectique entre la préservation et la destruction (l'un des thèmes les plus importants de Farocki) qu'à la mémoire et à l'oubli. Ces œuvres rendent ainsi visibles les tensions que suscite l'évocation nostalgique de pratiques, d'outils et de lieux en rapport au travail manuel, autant d'éléments dépouillés de leur valeur d'usage dans certaines régions du monde, mais qui resurgissent ailleurs sous le préfixe «trans» (transgressif, transnational, transitionnel et transhistorique). Et ces tensions peuvent à leur tour traverser les limites et les frontières, elles peuvent revenir et renverser, perturber les catégories établies, et non des moindres: pensons à la catégorie moderne fondamentale que j’ai évoquée, l'«auto-réflexion». Un «retour à l'œuvre» (un retour au travail) signifie donc que l'art qualifié «d'installation» doit non seulement demeurer auto-réflexif et auto-référentiel, mais aussi qu'il doit « performer » comme tel (autant de preuves de responsabilité réflexive, d'implication et de référence) : il doit retrouver la crisis dans le terme critique.

J'aimerais à présent préciser ce que j’entends par là. Premièrement, les films et les installations de Farocki sont critiques, surtout parce qu'ils remettent en cause la relation actuelle entre l'art et le travail, et donc notre conception de l'œuvre d'art. Nous pourrions définir l'art comme une association particulière entre l'œil et la main, entre voir et faire, une manière particulière d'engager le corps dans un geste d'auto-production ou d'auto-nomie au travers d'une symbolisation réflexive, ce qui implique aussi toujours la possibilité de la perte de soi (l'envers du mouvement vers l'«impersonnalité » de l'artiste moderne). Et nous pourrions parallèlement définir l'œeuvre (work) comme une association particulière entre l'œil et la main, dans un geste d'auto-objectivation qui implique aussi la possibilité de l'auto-aliénation, dans la rencontre du monde comme un tissu (fabric, tissue) ou un filet résilient de forces institutionnelles. Ces institutions règlent et régulent les formes de contrainte, de pouvoir et de rationalité que la technologie, l'industrialisation, l'État et le capitalisme produisent - cette

1. Un bref curriculum vitae, une filmographie complète ainsi qu'une liste de références bibliographiques peuvent être trouvés sur le site www.farocki-film.de. 
Fabrik que nous connaissons depuis deux siècles et demi, mais qui, comme nous le savons bien, s'ajuste et s'assouplit selon une variété de structures. L'indice ou le cluster métaphorique principal de cette conjonction œuvre/travail/art dans les films et les installations de Farocki est le caractère central du complexe main-œil/ main-machine/œil-machine.

Deuxièmement, et à la lumière de ce que j’ai avancé jusqu'ici, il est ici question d'«obsolescence transgressive », ce qui renvoie à la dialectique entre la mémoire et l'oubli, et surtout à la réappropriation par Farocki du complexe art/ aura/perte d'aura. Les théories de Walter Benjamin sur la relation entre art et travail (et particulièrement son hypothèse selon laquelle le désintérêt kantien envers l'objet artistique ne tiendrait qu'au souvenir de l'utilité préalable de celui-ci, dans un autre lieu et pour une autre communauté) ont été élaborées sous la double influence du surréalisme et du montage cinématographique russe. Ce qui lie l'art et le travail, selon Benjamin, c'est la sécularisation, le processus qui transforme les objets de culte religieux en œuvres d'art. L'évocation ironique, réflexive ou nostalgique de l'obsolescence des savoir-faire traditionnels a, depuis le début du $20^{\mathrm{e}}$ siècle, constitué la majorité des ressources et des matériaux à la base de ce que nous considérons être de l'art, et ce en réaction à l'acuité avec laquelle l'industrialisation a bouleversé le travail manuel organisé. Tel est l'héritage du surréalisme et de Marcel Duchamp, du pop art et de l'art conceptuel, que ce soit sous la forme de l'arte povera, du minimalisme ou d'une large part de l'art contemporain de l'installation. Selon ce contexte historique, les «images» que nous voyons aujourd'hui au musée (que ce soient des images techniques, peintes ou reproduites mécaniquement) sont envisagées comme des ready-made ou des objets trouvés plutôt que comme des «représentations» ou des actes mimétiques. La «matérialité » de l'image comme moyen d'expression conditionne sa valeur-signe, et celle-là dépend du support matériel dans lequel s'inscrit l'histoire ou l'archéologie d'un moyen de communication. Ainsi, l'image animée comme «objet trouvé» montre bien une nouvelle distribution des rôles, à la suite de la rencontre du monde de l'art avec la photographie, le film et la vidéo: les musées reconnus doivent se distinguer non plus par leur collection, mais par leur architecture; ils doivent rivaliser avec le design intérieur, la mode et les centres commerciaux pour capter l'attention du public; et leur finalité semble se résumer dans l'entreposage de technologies désuètes, comme c'est le cas pour la préservation des vidéos d'art et des films en $16 \mathrm{~mm}$ (un transfert en format DVD ne représente nullement une solution, n'importe quel commissaire ou historien de l'art pourra le confirmer). Mais en vertu de leur popularité, les musées sont des lieux de rencontres, de discussions et d'événements. Héritiers de l'époque 
bourgeoise, les musées doivent conserver leur rôle traditionnel d'intervenant culturel dans la cité conçue comme forum et agora. Par conséquent, l'artiste doit subir la tension d'être ou bien un designer de modes de vie et un propagateur de tendances, ou bien le gardien et l'archiviste d'outils et de techniques artisanales tombées en désuétude. Bien au fait de ce dilemme, Farocki a, selon moi, trouvé une autre avenue: se faire le gardien du travail et de l'œuvre, non par l'évocation de techniques obsolètes, mais plutôt par un engagement singulier envers les technologies de pointe qu'utilisent l'industrie, l'armée et même la gestion entrepreneuriale.

Troisièmement, je crois que l'art et le travail chez Farocki sont des activités similaires de séparation et d'assemblage - de l'art du tissage ou de l'art de couper du boucher jusqu'à la ligne de montage ou aux relais électroniques de l'ordinateur. Les films de Farocki (d'où sont issus ces exemples²) font paradigme à cet égard: ils dépeignent sans cesse des actions de séparation et de montage, d'assemblage et de désassemblage, de tricot et de décollage. Wie man sieht (Tel quion le voit, 1986) constitue tout un catalogue de ces actions, mais on les retrouve aussi dans tous ses films. Le corrélat rhétorique et l'incarnation esthétique d'une telle action de séparation et d'assemblage se retrouvent bien sûr dans la technique du montage, et donc dans cette dette de Benjamin envers le cinéma russe et Bertolt Brecht: se séparer de ce qui semble trop évident, d'un lieu commun ou d'un certain naturel, mais aussi forger des liens là où il n'y en a aucun ou rendre visible des liens invisibles, comme entre les prisons de haute sécurité et les centres commerciaux, ou entre l'industrie sidérurgique et le film indépendant. En suivant les propositions de Farocki, nous pourrions affirmer que l'art aujourd'hui serait en toute chose qui a subi un déplacement (ou un saut temporel) et dont la présence en un lieu ou en un moment donné constitue à la fois une rupture et un ré-assemblage, ainsi que la révélation rétrospective d'une ligne de force qui pointe vers un autre vecteur ${ }^{3}$.

Enfin, en raison de ce lien étroit mais voilé entre l'art et le travail, le principal motif de l'art moderniste (celui d'une auto-réflexivité, d'une réflexivité envers la matérialité et la dématérialisation et qui porte sur des conditions de possibilité et sur une impossibilité intrinsèque) ne peut s'arrêter à l'autoréférentialité. Comme j’ai déjà tenté de le montrer, une exigence éthique est implicite dans les films de Farocki, une exigence pour laquelle chaque acte

2. Tout ces exemples sont tirés de Wie man sieht (Tel qu'on le voit).

3. En ce qui a trait à cette conception de l'art, voir Jacques Rancière, Malaise dans l'esthétique, Paris, Galilée, coll. «La philosophie en effet», 2004, p. 160. 
auto-réflexif doit contenir une forme d'auto-responsabilité et même d'auto-implication. C'est dans cette prise de position ("mettre sa vie en jeu », comme Farocki l'écrivit dans une notice nécrologique pour son ami Holger Meins) que la crisis-critique, et donc la nature politique de l'art, particulièrement celle du film, se manifeste ${ }^{4}$.

Bien avant la plupart de ses contemporains, Farocki a constaté que les films industriels, les vidéos de formation, les bandes de surveillance, les études des temps et mouvements, les tomodensitogrammes des diagnostics médicaux et le design architectural assisté par ordinateur prenaient de plus en plus de place dans la culture visuelle. La persistance de ces « images opérationnelles » (Vilem Flusser) ou de ces «images techniques » (Friedrich Kittler5) juxtaposées aux travaux de Fritz Lang, d'Orson Welles, de Robert Bresson ou de Jean-Luc Godard (par exemple) a inspiré certaines des idées les plus pénétrantes sur le cinéma, dans les films de Farocki. Si Farocki parle d'aéroports, d'écoles et d'usines qui appartiennent à l'histoire de la vision et de la surveillance, dans Ich glaubte Gefangene zu sehen (Je croyais voir des prisonniers, 2000), il délimite aussi une possible post-histoire du cinéma; mais déjà dans Wie man sieht il avait tracé la pré-histoire de l'ordinateur en soulignant comment la bifurcation d'une route ou l'affluent d'un ruisseau qui rejoint une rivière avait mené à la fondation de villes, et il avait encore démontré combien le principe de séparation/assemblage est fondamental à l'habitation humaine, tout comme le si/alors, le oui/non, les arborescences informatiques et celles de la narrativité contemporaine. Wie man sieht explique aussi comment le métier à tisser Jacquard et sa séquence de fils colorés programmable a «anticipé » la télévision, ou pourquoi le déploiement de la mitrailleuse Maxim lors de la bataille d'Omdurman a été une étape nécessaire dans l'«invention » du cinématographe des frères Lumière. En ce sens, le cinéma de Farocki est un méta-cinéma : il dépasse le cinéma qui nous est familier, en réfléchissant au cinéma qui nous est inconnu.

Les films de Farocki sont en constant dialogue avec les images et la fabrication d'images, mais ils ont aussi toujours considéré les institutions qui les produisent

4. Harun Farocki, «Sein Leben einsetzen. Bilder von Holger Meins», Jungle World Supplement, 23 décembre 1998. Pour la traduction anglaise «Staking One’s Life», Harun Farocki. Working on the Sight-Lines, Thomas Elsaesser (dir.), Amsterdam, Amsterdam University Press, 2004, p. 83-94.

5. Voir Andreas Ströhl, (ed.), Vilem Flusser: Writings, Minneapolis, University of Minnesota Press, 2004, Vilem Flusser, Ins Universum der technischen Bilder, Göttingen, 1999 et Friedrich Kittler, Draculas Vermächtnis Technische Schriften, Leipzig, Reclam Verlag, 1993. 
et les propriétaires qui les font circuler (ou les suppriment), comme l'industrie du film, la publicité, la télévision, l’armée, le gouvernement. Ceci fait aussi partie de son méta-cinéma présenté «à neuf» au Musée - une institution qui, même en paraissant opérer «en dehors» des contraintes institutionnelles tout juste mentionnées, ne «communique» pas moins (comme un vase communicant) avec elles dans une réciprocité clandestine. Comme l’a écrit Farocki : «Mes films sont faits contre le cinéma et contre la télévision », une phrase qui porte encore l'écho d'une rhétorique de protestation et de confrontation post-soixante-huitarde, et semble maintenant pointer vers le «cube blanc» de l'espace en galerie, tout en reconnaissant le fait fondamental qu'il n'y a plus d'espace «hors» des images et de leur fonction politique ou institutionnelle, plus d'espace à partir duquel parler des images. Pourtant son méta-cinéma fonctionne sans méta-langage: dans ses films pour la télévision qui s'opposent à la façon dont la télévision fait des films, «l'intérieur est l'extérieur est l'intérieur». Betrogen (Trahi, 1985) constitue un bon exemple: jouer de manière décisive un soap-opera contre un thriller, dans un jeu de coups et de contre-coups qui révèlent autant de couches d'illusion ou de délire, comme si un genre était le «double-fond» de l'autre, dans une lutte entre ce que Deleuze nomme «les puissances du faux ${ }^{6} »$. Ou pour le dire autrement: tout comme le cinéaste est impliqué dans la machinerie de l'industrie cinématographique, en dépit de ses efforts pour s'en extirper, ses images font partie de la construction-du-monde que produisent les caméras; par conséquent, une telle construction-du-monde est aussi une perte-du-monde. Comme le disait Farocki en entrevue avec moi, son cinéma contribue lui aussi à rendre ce monde superflu, aux côtés des satellites qui patrouillent le ciel et des caméras en continu de CNN et consort?. Dans la dialectique de la «capture » et de «l'entreposage », de la «couverture» et de «l'enregistrement», l'image animée se donne le statut de «mémoire collective » et accumule son capital culturel en dévaluant ce qu'elle montre, que son geste soit de "préservation» ou de «rédemption », de «révélation» ou de «pure visibilité »; les actes de préservation du cinéma sont des actes de destruction, et la mémoire s'enchevêtre avec l'oubli, dans le ruban de Moebius d'une implication réciproque.

Parmi ces gestes ambigus, le concept de travail intervient par l'entremise d'un signifiant métonymique, la main. Farocki a souvent comparé la création

6. Gilles Deleuze, L'image-temps, Paris, Les Éditions de Minuit, coll. «Critique», 1985, p. 172.

7. «Making the World Superfluous: An Interview with Harun Farocki», Harun Farocki. Working on the Sight-Lines, Thomas Elsaesser (dir.), p. 177-192. 
de films à une forme d'écriture (notamment dans Schnittstelle [Section, 1995], (fig. 1), aussi l'étiquette de «film-essai » décrit-elle bien un aspect important de son travail, mais seulement si on entend aussi par là une référence à la racine étymologique du mot essai (qui remonte au verbe faire), en plus d'une référence à la «manu-facture» de Farocki, à son écriture manuscrite, sa signature. En d'autres mots, la métaphore utilisée par Benjamin pour décrire la présence indirecte du narrateur dans le récit, «l'empreinte digitale du potier sur le récipient d'argile», s'applique aussi à ce «film-essai » ${ }^{8}$. C'est bien connu, dans plusieurs des films de Farocki, la main du réalisateur cadre une image dans le film (de manière remarquable dans Bilder der Welt und Inschrift des Krieges [Images du monde et inscription de la guerre, 1988] et Schnittstelle).

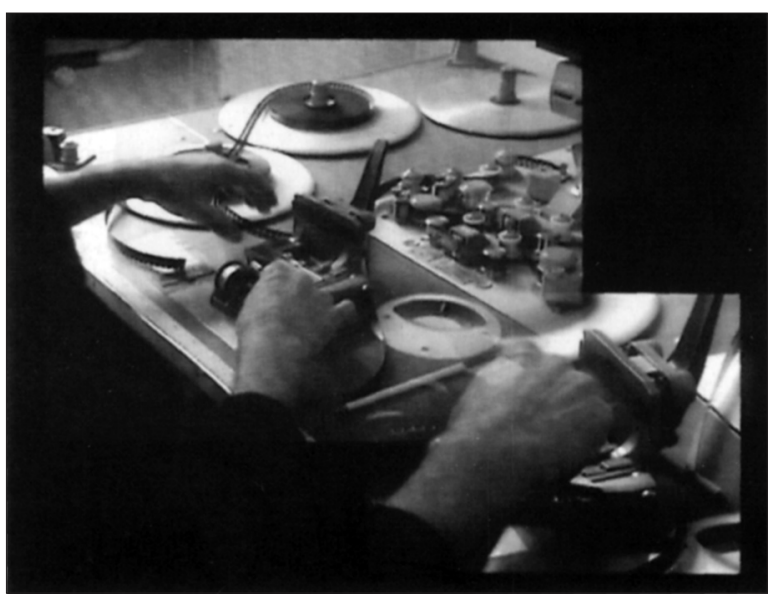

Fig. 1: Harun Farocki, Schnittstelle/Section, 1995.

En collaboration avec Jürgen Becker, Farocki a aussi tourné un film simplement intitulé Der Ausdruck der Hände [L'expression des mains, 1997], (fig. 2). Il s'agit d'un film qui célèbre la grâce et la finesse des mains humaines, instruments du toucher et de la caresse, moyens du don et de la préhension. Mais on y montre aussi les mains au travail : elles sont les outils chirurgicaux du voleur à la tire - ou encore elles manipulent du matériel et des machines dans le processus de production manufacturière. On éprouve aussi dans ces chorégraphies de

8. Voir à ce sujet, Walter Benjamin, «Le conteur. Réflexions sur l'œuvre de Nicolas Leskov [1936]», dans Euvres complètes, tome III, Paris, Gallimard, coll. « Folio essais », 2000 , p. $114-151$. 


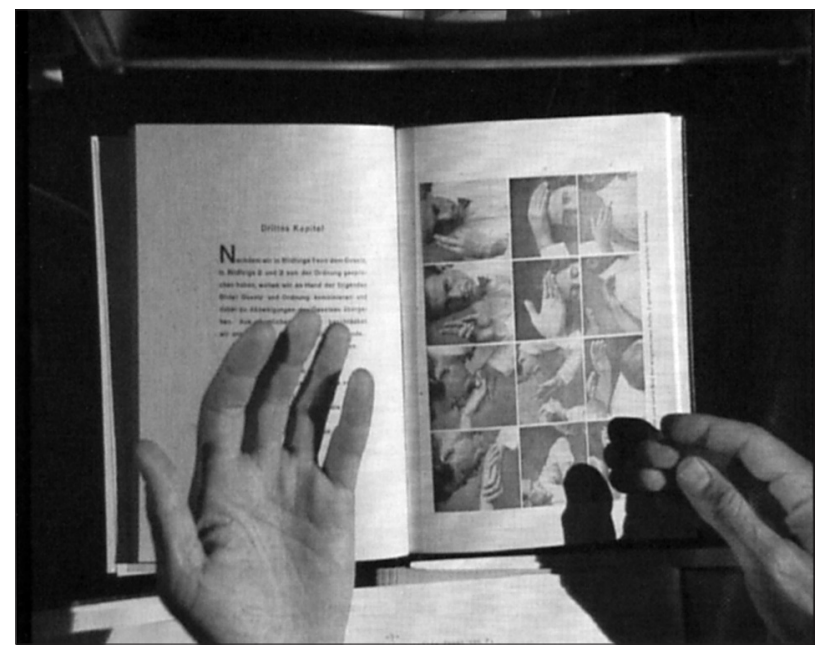

Fig. 2: Harun Farocki, Der Ausdruck der Hände/L'expression des mains, 1997 .

doigts agiles, de paumes puissantes jusqu'au bout délicat des doigts, la sensation que la célébration tient du requiem, comme chez Henri Focillon dans son éloge de la main, écrit alors qu'il s'apprêtait à passer à la machine à écrire9. Cela rappelle encore ce que j’évoquais précédemment, à savoir que la première moitié du $20^{\mathrm{e}}$ siècle peut être rétrospectivement envisagée sous l'angle de l'histoire, brève et vite oubliée, de l'obsolescence de la main dans les processus de production. Tandis que la force de la main était remplacée par la machine, les fonctions de guidage et de conduite de la main ont été attribuées à l'œil, lequel surveille et contrôle les processus de production accomplis par des machinesoutils informatisées, par des bras robotiques et des pinces en métal au lieu de poignets et de doigts - du moins en Occident: les mains des femmes dans les chaînes de montage d'Asie racontent la même histoire géopolitique au ralenti. Une structure qui manufacture en théorie des briques à Mumbai et au Burkina Faso, mais qui les produit à la machine en France et en Allemagne, une telle structure représente bien plusieurs a-synchronicités et hiatus, ces a-symétries et ces faux parallèles qui sont lancés dans une juxtaposition et une combinaison simples, lesquelles ne sont, encore une fois, que des séparations et des assemblages, le rassemblement de différents sites de production et de différents processus manufacturiers imitant les diverses étapes de la fabrication de briques, et

9. Henri Focillon, Vie des formes, suivi de Éloge de la main [1943], Paris, Presses universitaires de France, 1981, p. 101-128. 
vice versa. Les éléments distincts, les outils et les compétences requis dans la fabrication de briques, reflètent chacune des étapes de la production de films en celluloïd, une analogie qui ramène au souvenir ironique des célèbres théories du langage filmique de Koulechov et Poudovkine, qui comparaient le montage d'une séquence visuelle, plan par plan, à la construction d'un mur, brique par brique.

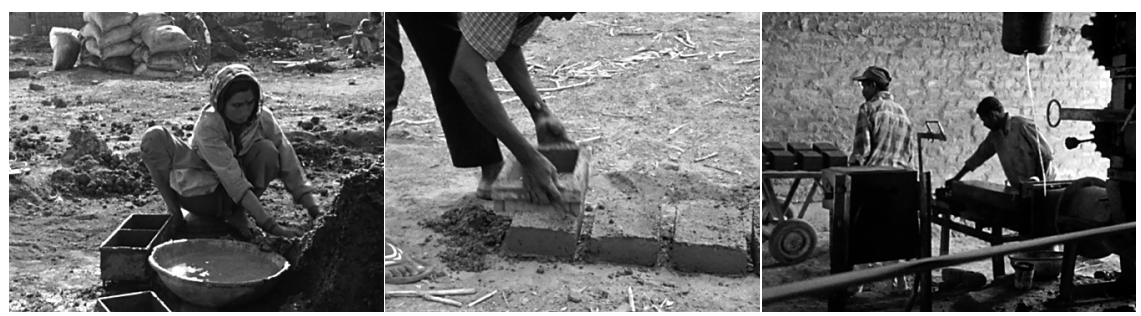

Fig. 3 a,b,c: Harun Farocki, Vergleich über ein Drittes/Comparaison via un tiers, 2007.

Je fais bien entendu référence à Vergleich über ein Drittes/Comparaison via un tiers (2007, partie de Nebeneinander/Côte à côte, une présentation de son travail à Vienne). Dans cette œuvre, la conception de la manu-facture, de «l'œuvre-à-la-main » et de sa situation historique (à la fois avant-garde en esthétique et obsolescence en industrie), se pose pour Farocki selon la question du genre d'« œuvre» (work) qu'est aujourd'hui devenue la création filmique, particulièrement la sienne. Parce qu'il semble (du moins pour moi) que ce que Farocki désigne par l'énigmatique das Dritte (le tiers) n'est finalement rien d'autre que la création filmique elle-même, comparée et enveloppée dans le Nebeneinander (le côte à côte)de processus manufacturiers juxtaposés. Dans l'installation que j’ai visitée à Vienne, le cinéma était implicitement invoqué dans sa mise en scène, son souvenir, son écho sur la bande son (qui reproduit le bruit d'un projecteur), alors qu'il était élidé dans l'image - à l'exception du lien métaphorique que je viens de mentionner. Cela dit, ce qu'il faut remarquer, c'est qu'il y a plus d'un moment de réflexivité moderniste relayé par le sens métaphorique de la «brique» dans l'histoire de la théorie du cinéma ${ }^{10}$. La création de films mis en vedette au musée (sujette à la pleine tension de l'obsolescence technique devenue art noble), est peut-être la dernière sorte d'œuvre ou de travail qui mérite ce nom; elle sert d'allégorie pour un grand nombre de travaux et d'œu-

10. Voir par exemple, Jean Mitry, Esthétique et psychologie du cinéma, vol. I, Paris, Éditions universitaires, 1963 , p. 48 . 
vres sans nécessité ni valeur. Dans Etwas wird sichtbar [Le Vietnam nous appartient], Farocki se situe quelque part entre «travailler comme une machine» et «œuvrer comme un artiste», et il juge ces deux positions trop faciles: «Il ne s'agit pas de faire l'une ou l'autre, mais de joindre les deux. » Cependant, bien qu'en 1981 une telle déclaration constituait un commentaire critique sur la création filmique «indépendante» en RFA, entre le déclin de l'industrie cinématographique et le marché croissant des artistes-artisans qui travaillaient pour la télévision, le dilemme main/œil/machine est depuis devenu partie prenante d'une réflexion plus large sur les «fins» (et bien sûr aussi, sur «la fin ») du cinéma, tel que cela s'annonçait aux débuts du cinéma, quand Eadweard Muybridge a fait entrer la chronophotographie dans les nouvelles lignes de montage industriel, pour mener des études des temps et mouvements.
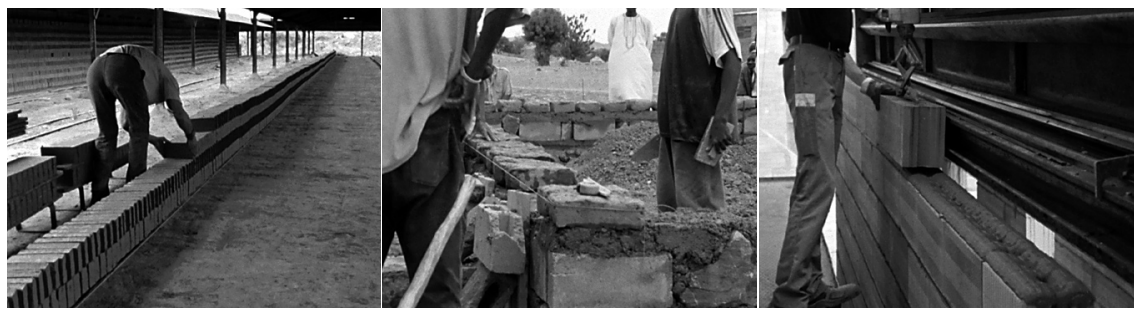

Fig. 4 a, b, c: Harun Farocki, Vergleich über ein Drittes/Comparaison via un tiers, 2007.

Une telle archéologie du cinéma, qui fait revenir le cinéma à ses origines d'une façon introspective et multiple, met aussi en lumière la signification particulière d'un film de Farocki réalisé en 1995 à l'occasion du centième anniversaire de «naissance» du cinéma: Arbeiter verlassen die Fabrik (La sortie des usines, 1995) sa propre version (et répétition) du célèbre film des frères Lumière, La sortie des usines Lumière (1895). Extension et enveloppe métaphorique de la fenêtre d'image (le défilén ), le sujet ironique (une autre "comparaison faite au moyen d'un troisième terme [caché]») de ce film de compilation (composé de matériel fictif et réel autour du trope/lieu de l'entrée de l'usine), c'est la convergence emblématique d'une technologie singulière, le cinématographe, et d'un lieu singulier, la manufacture. Une ironie supplémentaire réside dans le fait que, depuis la rencontre entre le cinématographe et l'usine, de plus en plus de travailleurs sont précisément «sortis de l'usine». Avec l'apparition du cinéma, 
et dans une large mesure en raison de celle-ci, paradoxalement, la valeur et la fonction du travail et de la créativité se sont considérablement transformées. Plusieurs sociétés occidentales semblent s'être figées face au chômage et au sousemploi, de plus en plus d'ordinateurs personnels (mutations technologiques du cinématographe) ont envahi les lieux de travail, et du même souffle le chez-soi s'est fait usine, et le loisir, travail: quelque part dans ces a-symétries réfléchies, le cinéma à la fois coupe et relie. Pour le dire autrement, selon Farocki, le cinéma est doublement impliqué dans une histoire - celle du travail - qui dépasse largement l'histoire du cinéma.

Les films et les installations de Farocki ont donc depuis les années 1990 porté de plus en plus sur la question du «travail », et d'une façon qui dépasse les catégories du monde économique, c'est-à-dire la manière dont une société produit matériellement et idéologiquement les moyens de sa survie. Lavenir du travail est un point encore plus décisif, parce qu'il concerne ce que signifie être (et demeurer) humain. Et c'est ici que Farocki remarque, du haut de son poste d'observation en galerie, point de vue idéal pour mener une telle introspection, le rôle fatal que le cinéma a peut-être joué - lorsqu'il parle de la filiation qui lie les premières études des temps et mouvements avec les satellites de surveillance dernier cri. Je cite: «Si les études des temps et mouvements de l'usine fordiste dépeignaient un travail abstrait, les images des caméras de surveillance laissent voir une existence abstraite ${ }^{12}$.»

Mais alors, quelle est la fonction de l'œil dans cette implication du cinéma dans l'émergence d'une existence abstraite? Si la machine mécanique a pu rendre la main obsolète au cours de la première moitié du $20^{\mathrm{e}}$ siècle, les machines de vision électroniques n'ont-elles pas rendu l'œil superflu dans la dernière moitié du siècle? Ces machines ne sont pas fondées sur l'optique telle que nous la connaissons depuis Descartes ou Newton : elles génèrent plutôt des «données » (variations de courant électrique, changements de température, fluctuations de la densité atmosphérique...) que les algorithmes d'un ordinateur convertissent en visualisations ou en représentations graphiques que nous appelons encore, par habitude, des «images». Cette question est importante, non seulement parce qu'une grande part de la «vie » semble avalée par de telles images, par leur séduisante allure colorée, sous la forme de graphiques informatiques ou d'effets spéciaux, mais aussi en raison de la fonctionnalité instrumentale des images (tant dans une exploitation industrielle que dans une application médicale) tel

12. Harun Farocki, «Controlling Observation », in Harun Farocki. Working on the SightLines, Thomas Elsaesser (dir), p. 293. Publié dans Jungle World le 8 septembre 1999. 
que démontré dans Gegen-Musik (Contre-chant, 2004). Là où les théoriciens du cinéma ont exploré les dialectiques de la vision incarnée et désincarnée et se sont inquiétés des conséquences troublantes d'un concept de «vision » (look) et de «regard» (gaze), les films de Farocki ajoutent une dimension: celle de l'œil machinique, avec son regard aveugle, avec les ruptures qu'un nouveau régime de surveillance omniprésente engendrent dans l'œil/je, dans les relations sujet/objet qu'implique une optique cartésienne. Le fait que beaucoup de films de Farocki portent un titre comportant les mots «voir» ou « œil » (Wie man sieht [Tel qu'on le voit]; Etwas wird sichtbar; Auge/Maschine [Eil/Machine (200o)]; Ich glaubte Gefangene zu sehen [Je croyais voir des prisonniers]) nous rend attentifs aux paradoxes mortels de la vision et de la visualité en rapport avec la représentation, la surveillance et le sujet voyant. Encore une fois, il n'y a pas de dehors à cet intérieur : le regard (look) du cinéaste - qui englobe la [subjectivité chaude de la] vision de l'artiste et l'[œil froid du] regard de la machine - est aussi le regard de la Gorgone : il pétrifie ce qu'il voit, il rend les images temporaires et les transforme en objets, en échantillons, en pièce exposée.

Si on tient compte de cette fatalité du cinéma (même lorsqu'il intègre des applications scientifiques, militaires, industrielles et médicales comme chez Farocki), que peut faire le cinéaste «post-avant-gardiste»? Opérer dans les zones liminaires de son métier, un métier qui a cependant migré dans l'espace culturellement central du musée, et devenir «hors-territoire» et se marginaliser en considération de l'espace public qu'était naguère le cinéma? Farocki pratique un regard plus sceptique, plus médicolégal, à l'encontre de cette nostalgie de la préservation et de la rédemption, ou encore de l'utopie de la révélation et du dévoilement traditionnellement associés à l'image photographique dite «artistique ». Toutefois, à la différence du protagoniste photographe de Blow Up (Michelangelo Antonioni, 1966) qui s'enfonce à l'intérieur d'une unique image pour y extraire une preuve, la méthode de Farocki est bien singulière : assembler et séparer des images, juxtaposer deux images, exprimer une image au travers d'une seconde image ou aligner une série d'images, découvrir des motifs significatifs. Nulle part n'est-ce aussi évident que dans Bilder der Welt und Inschrift des Krieges, dont le cour tient en deux séries de photographies de 1943/44: l'une d'elle prise par les avions de reconnaissance américains survolant Auschwitz, sans voir «Auschwitz», et l'autre prise par un officier SS «au sol » soucieux de placer dans son album de photos le processus de sélection ayant lieu sur la rampe du camp de concentration, sans paraître remarquer ni la cruauté innommable de cet instant ni la bouleversante humanité qui le regarde et regarde au travers de la lentille. Ces deux regards ont ainsi deux sortes de froideur, et ils ne libèrent 
leurs propres déterminations historiques et leurs significations éthiques qu'en entrant en collision. La tragédie tient dans un écart béant et un non-vu que seulement l'assemblage, la répétition et le montage, que ce soit dans le transfert, dans la translation ou dans la substitution, peuvent consigner et enregistrer pour une diffusion ultérieure, une propagation dans l'aveniri' ${ }^{13}$. L'œeil froid» cède la place à la «main» comme instrument de montage, lequel ouvre à son tour la séquence d'un œil intérieur qui n'est plus séduit ou trompé par la «vision» : les principes de Vertov adaptés à une nouvelle génération (de machine de vision). En d'autres termes, dans l'acte métaphorique d'assembler se trouve parfois dissimulé le tertium comparationis ( Vergleich über ein Drittes») qui ne se montre que dans le résultat métonymique de la séparation par la contiguïté, ce changement de lieu et (ou) de temps qui m’a servi à définir précédemment l'«art». Le déplacement et les sauts temporels engendrent ainsi les coordonnées complexes d'une «poétique de la métaphore» qui doit être considérée comme le geste qui fonde les méthodes de travail de Farocki, tel qu'il l'explique lui-même dans Schnittstelle mais ils déterminent aussi la logique qui amène le travail de Farocki au musée.

Ce sont donc deux sortes de réseaux qui déplacent le cinéma vers le musée, dans l'œuvre de Farocki. Le premier est «interne» et désigne la «poétique de la métaphore» et la «politique de la métonymie» décrites ci-dessus. Le second est «externe» et relie les différentes institutions qui actuellement produisent, promeuvent ou préservent l'image animée : la télévision, le cinéma, le monde de l'art, les archives, les bases de données visuelles, en plus des autres usagers institutionnels des secteurs de la surveillance et de la sécurité, l'armée, le commerce et l'industrie, lesquels emploient l'image à des fins d'entraînement, de test ou de contrôle. Chacun est non seulement en mouvement par rapport aux autres, mais est aussi a-symétrique et donc marqué de moments d'échanges inégaux et de rapports de pouvoir structurels, et parfois suturé par un terme caché ou invisible - ce que Farocki expose avec zèle mais délicatesse.

Ceci s'observe clairement dans des installations de Farocki comme Die Schöpfer der Einkaufswelten [Les créateurs des mondes de consommation, 2001], (fig. 5) ou dans la série Auge/Maschine (2001-2002). Par exemple, rapprocher des prisons et des centres commerciaux vise à provoquer une réflexion sur le tertium comparationis d'une façon qui diffère en l'occurrence de la comparaison entre

13. Voir à ce sujet, Kaja Silverman, «What is a Camera?, Discourse, n ${ }^{\circ}$ 15, printemps 1993, p. 3-56; Nora M. Alter, «The Political Imperceptible in the Essay Film: Farocki's Images of the World and the Inscription of War », New German Critique, n 68, printemps-été 1996, p. 165-192. 


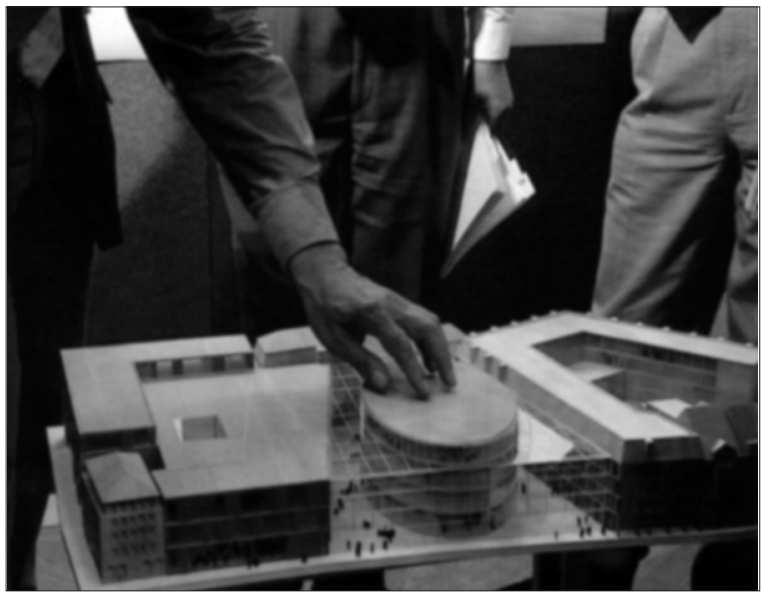

Fig.5: Die Schöpfer der Einkaufswelten/Les créateurs des mondes de consommation, 2001.

la "production» de cadavres dans les tranchées de la Première Guerre mondiale et la production de voitures dans les lignes de montages fordistes (une analogie faite par Farocki dans Zwischen den Kriegen [Entre deux guerres, 1977-78] ). Parce que certaines analogies ne peuvent plus être pleinement perçues (on pourrait se demander ce qui peut bien lier la vidéo d'une caméra de surveillance prise durant la visite guidée d'une prison avec l'image granuleuse de clients qui poussent des paniers dans un supermarché), il faut déconstruire les comparaisons. La prison panoptique que montre Farocki dans la scène d'ouverture de Ich glaubte Gefangene zu sehen, avec ses rangées alignées de caméras et de mires, est déjà obsolète (comme le remarque lui-même Farocki) en regard des nouvelles technologies de surveillance «déterritorialisée » qui permettent le marquage et le retraçage. D'une manière analogue, la vente au détail est devenue un exercice raffiné de marquage, de retraçage et de profilage à l'aide de bases de données, tout comme le soccer (ou football) pour le jeu des commentateurs qui observent les joueurs - si nous nous en tenons à Deep Play de Farocki, une installation présentée à Kassel en 2007, à l'occasion de documenta 12. L'un des objectifs de Farocki, sous cette pléthore de graphiques, de vecteurs et de traitements de données en temps réel, est d'exposer les limites mêmes du visible en regard des industries à forte marge commerciale mais au profil politique bas (puisqu'elles visent des économies d'échelle), lesquelles sont apparues à la faveur d'alliances forgées entre les firmes de logiciels, les laboratoires universitaires, les spécialistes en sécurité, les fédérations sportives et les industries du service au consomma- 
teur : un nouveau complexe militaro-récréatif qui a depuis longtemps supplanté l'ancien complexe militaro-industriel.

Ce qui m’amène à mon dernier point: la traditionnelle auto-référentialité du musée comme lieu d'une nouvelle responsabilité et d'une nouvelle implication du cinéaste. La tendance générale à des processus d'autorégulation automatisés et à l'usage de systèmes de rétroaction compliqués dans nos organisations sociales, notre concept de «vie artificielle » et aussi notre art d'avant-garde soulignent l'actualité du travail de Farocki. On pourrait avoir l'impression que Farocki ne suit que l'actualité médiatique (par un rapprochement entre le Vietnam et les années 1970; Auschwitz et les années 1980; les technologies de surveillance, les bombes intelligentes et les années 1990; les régimes carcéraux, les centres commerciaux, les supermarchés et les années 2000), mais cette impression est erronée, voire trompeuse. La contemporanéité de Farocki dépasse la simple volatilité des manchettes : elle se place au cœur de sa vision politique, qui demande à être témoin du présent. Qu'est-ce que cela signifie en pratique? Farocki ne choisit un sujet que lorsque celui-ci peut ouvrir une mise en abyme, en tant qu'image/inscription du monde qui reflète aussi le travail et les méthodes de Farocki : c'est un système de rétroaction qui est toutefois asymétrique et asynchrone, au lieu d'être fermé et autorégulé. C'est par exemple le cas des deux séries de photographies de Bilder der Welt, du mouvement de va-et-vient entre le bureau et la table de montage dans Schnittstelle, mais aussi, comme j'ai tenté de le montrer, celui d'installations plus récentes, comme Nebeneinander. L'installation, comme double mouvement de correspondance conjointe et de disjonction, de face et de retour, semble être le point de référence implicite d'une entreprise qui a commencé bien avant que le cinéma de Farocki n’entre dans une galerie d'art; en fait, cela remonte à l'un de ses premiers films, Nicht löschbare Feuer [Feu inextinguible, 1969]. Il existe une phrase en allemand pour signifier un engagement total et une responsabilité entière: seine Hand ins Feuer legen, se mettre la main au feu. Ne s'agit-il pas du genre d'engagement que prend Farocki dans Nicht löschbare Feuer?

En résumé, les films de Farocki ont une autorité morale et une crédibilité esthétique précisément dans la mesure où leur réflexion sur la surveillance et l'existence abstraite constitue une lame à double tranchant: d'une part, envers le cinéaste lui-même, et d'autre part en ce que la boucle de rétroaction implique l'artiste comme une personne capable de mettre sa caméra en jeu. C'est ainsi que Farocki est peut-être l'un des seuls cinéastes aptes à briser réflexivement l'autosurveillance du monde comme œil machinique. La véritable actualité, la véritable urgence de son œuvre d'installation ne tient peut-être nulle part ailleurs que 
dans son effort pour sauver le monde de sa propre superficialité, quand celui-ci se reflète dans l'efficacité automatisée de ses images opérationnelles. En dé-faisant méticuleusement ces images, non tant en objets trouvés destinés à un musée du futur, mais en les appelant comme la mémoire de corps en mouvement et comme la pensée en action, les installations de Farocki invitent le visiteur à imaginer à nouveau le musée ou la galerie comme le lieu d'une rencontre publique et d'un événement politique, même s'il s'agit d'un espace qui demeure consacré à l'art.

Traduit de l'anglais par Patrick Poulin. 